\title{
Avaliação radiográfica do alinhamento pós-operatório na artroplastia total de joelho*
}

\section{Radiological Evaluation of Postoperative Alignment in Total Knee Arthroplasty}

\author{
Leonardo Dalla Giacomassa Rocha Thomaz ${ }^{10}$ João Guilherme Brochado Geist² ${ }^{20}$ \\ Rafael De Luca De Lucena ${ }^{3(0)}$ Carlos Roberto Schwartsmann ${ }^{4,5}$ Geraldo Luiz Schuck de Freitas ${ }^{6(0)}$ \\ Leandro de Freitas Spinelli $7,8,9$ (1)
}

1 Serviço de Ortopedia e Traumatologia, Santa Casa de Misericórdia de Porto Alegre, Porto Alegre, RS, Brasil

2 Serviço de Ortopedia e Traumatologia, Hospital Regina, Novo Hamburgo, Porto Alegre, RS, Brasil

3 Serviço de Ortopedia e Traumatologia, Santa Casa de Misericórdia de Porto Alegre, Porto Alegre, RS, Brasil

${ }^{4}$ Departamento de Ortopedia e Traumatologia, Universidade Federal de Ciências da Saúde de Porto Alegre, Porto Alegre, RS, Brasil

${ }^{5}$ Serviço de Ortopedia e Traumatologia, Complexo Hospitalar Santa Casa de Misericórdia de Porto Alegre, Porto Alegre, RS, Brasil

${ }^{6}$ Grupo do Joelho, Santa Casa de Misericórdia de Porto Alegre, Porto Alegre, RS, Brasil

${ }^{7}$ Faculdade de Medicina, Universidade Federal de Ciências da Saúde de Porto Alegre, Porto Alegre, RS, Brasil
Endereço para correspondência Leonardo Dalla Giacomassa Rocha Thomaz, MD, Serviço de Ortopedia e Traumatologia, Santa Casa de Misericórdia de Porto Alegre, Rua Professor Annes Dias, $135 / 2^{\circ}$ andar, Centro Histórico, Porto Alegre, RS, 90460-150, Brasil

(e-mail: leorochat@hotmail.com).

\footnotetext{
${ }^{8}$ Grupo de Cirurgia do Quadril, Serviço de Ortopedia e Traumatologia, Complexo Hospitalar Santa Casa de Porto Alegre, Porto Alegre, RS, Brasil

${ }^{9}$ Laboratório de Bioengenharia, Biomecânica e Biomateriais, Programa de Pós-Graduação em Projeto e Processos de Fabricação, Universidade de Passo Fundo, RS, Brasil
}

\section{Resumo \\ Palavras-chave \\ - artroplastia do joelho \\ - articulação do joelho \\ - radiografia}

Objetivo Demonstrar a maior acurácia das radiografias panorâmicas de membros inferiores (longas) em relação às radiografias curtas do joelho na medida do eixo mecânico do membro inferior após a artroplastia total de joelho (ATJ).

Métodos Foi realizado um estudo retrospectivo para avaliar a acurácia de imagens radiográficas longas e curtas pós-operatórias de 70 pacientes submetidos à ATJ em nosso serviço. As imagens foram analisadas ao acaso, em momentos distintos, por três ortopedistas. Em todas as imagens, o eixo mecânico do membro, do fêmur e da tíbia foram traçado,s e os ângulos femorotibiais (AFTs) foram calculados. O coeficiente de correlação intraclasse (CCI) foi calculado para avaliar a concordância da medida do eixo mecânico inter e intraobservador.

\footnotetext{
Trabalho desenvolvido no Serviço de Ortopedia e Traumatologia da Santa Casa deMisericórdia de Porto Alegre e Universidade Federal de Ciências da Saúde de Porto Alegre, Rio Grande do Sul, Brasil.
}

recebido

15 de Agosto de 2020

aceito

28 de Outubro de 2020

Publicado on-line

Abril 19, 2021
DOI https://doi.org/

$10.1055 / \mathrm{s}-0041-1726061$

ISSN 0102-3616.

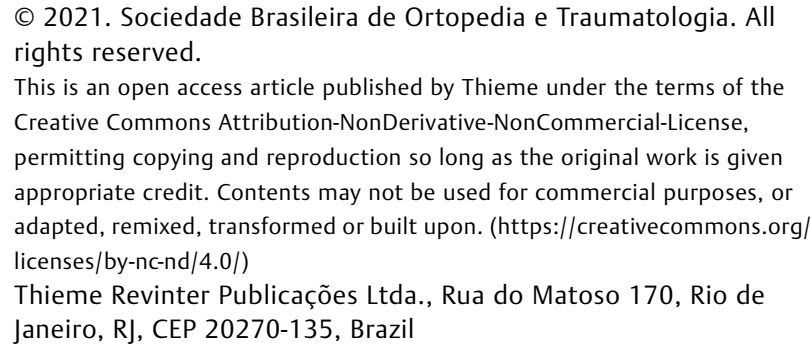

(c) 2021. Sociedade Brasileira de Ortopedia e Traumatologia. All rights reserved.

This is an open access article published by Thieme under the terms of the Creative Commons Attribution-NonDerivative-NonCommercial-License, permitting copying and reproduction so long as the original work is given appropriate credit. Contents may not be used for commercial purposes, or adapted, remixed, transformed or built upon. (https://creativecommons.org/ licenses/by-nc-nd/4.0/) Thieme Revinter Publicações Ltda., Rua do Matoso 170, Rio de Janeiro, RJ, CEP 20270-135, Brazil 


\section{Abstract}

\section{Keywords}

- arthroplasty, replacement, knee

- knee joint

- radiography
Resultados Observou-se que houve alta concordância intra e interobservador quando utilizamos radiografias panorâmicas, apresentando $\mathrm{CCI}$ mínimo intrae interobservador de 0,89 , equivalente a uma concordância fortíssima. Já nas radiografias curtas na incidência anteroposterior (AP) do joelho, o CCl mostrou-se com concordância moderada, obtendo valor máximo de 0,75 .

Conclusão Existe uma diferença significativa na acurácia para a medida do eixo mecânico do membro inferior, comparando-se radiografias longas e curtas do membro inferior. Assim, para a adequada mensuração do eixo mecânico do membro inferior, sugerimos a realização de radiografia longa no pós-operatório de AT].

Objective To demonstrate the greater accuracy of panoramic radiographs of the lower limbs (long) in relation to short radiographs of the knee in the measurement of the mechanical axis of the lower limb after total knee arthroplasty (TKA).

Methods A retrospective study was conducted to evaluate the accuracy of long and short postoperative radiographic images of 70 patients submitted to TKA in our service. The images were analyzed at random, at different times, by three orthopedists. In all images, the mechanical axis of the limb, femur and tibia were traced and femorotibial angles (FTAs) were calculated. The intraclass correlation coefficient (ICC) was calculated to evaluate the agreement of the measurement of the inter- and intraobserver mechanical axis.

Results It was observed that there was high intra and interobserver agreement when panoramic radiographs were used, with minimum intra and interobserver ICC of 0.89 , equivalent to a very strong agreement. On short radiographs in the anteroposterior incidence (AP) of the knee, the ICC showed moderate agreement, obtaining a maximum value of 0.75 .

Conclusion There is a significant difference in accuracy for the measurement of the mechanical axis of the lower limb, comparing long and short radiographs of the lower limb. Thus, for the proper measurement of the mechanical axis of the lower limb, we suggest the performance of long radiography in the postoperative period of TKA.

\section{Introdução}

A artroplastia total de joelho é uma cirurgia amplamente realizada em todo mundo, com altos índices de sucesso. Estima-se que nos EUA ocorra um crescimento da demanda cirúrgica deste procedimento de $673 \%$ (aproximadamente 3,48 milhões) entre o período de 2005 a $2030 .^{1}$ A avaliação pós-operatória cirúrgica envolve uma série de cuidados. Além do controle da ferida operatória, medidas de amplitude de movimento e avalição de escalas de dor, a avaliação pós-operatória do alinhamento radiográfico dos eixos mecânico e anatômico também é parte fundamental para o sucesso do procedimento. ${ }^{1-3}$

A radiografia panorâmica de membros inferiores tem se mostrado essencial no planejamento pré-operatório. Além da medida radiológica dos eixos, podem-se identificar possíveis deformidades femorais e tibiais que venham a dificultar o ato cirúrgico. ${ }^{4}$ Já as radiografias curtas na incidência anteroposterior (AP) e de perfil com apoio monopodálico, além da incidência de Rosenberg, são ótimas para estadiamento e classificação da patologia degenerativa.

Nas avaliações pós-operatórias, é extremamente comum encontrarmos apenas radiografias curtas do joelho, impos- sibilitando a comparação com o alinhamento pré-operatório e a identificação daqueles implantes que podem estar em risco de falha prematura. ${ }^{5}$ Muitos autores justificam a não realização das radiografias longas por aumentar os custos para o sistema de saúde, além do fato de o exame não estar disponível para realização em todos os centros de imagem.

Os trabalhos na literatura divergem em relação ao nível de concordância inter e intraobservador sobre o alinhamento mecânico e anatômico do membro inferior utilizando radiografias longas e curtas do joelho. ${ }^{6,7} \mathrm{O}$ objetivo deste estudo é comparar a acurácia intra e interobservador da avaliação do eixo mecânico entre radiografias panorâmicas de membros inferiores e radiografias de joelho no pós-operatório de pacientes submetidos a artroplastias totais do joelho (ATJs) realizadas no nosso serviço.

\section{Materiais e Métodos}

O presente estudo foi submetido e aprovado pelo Comitê de Ética em Pesquisa de nossa instituição sob o número CAAE 33420820.5.0000.5535. Foram analisadas retrospectivamente radiografias pós-operatórias de 70 pacientes 

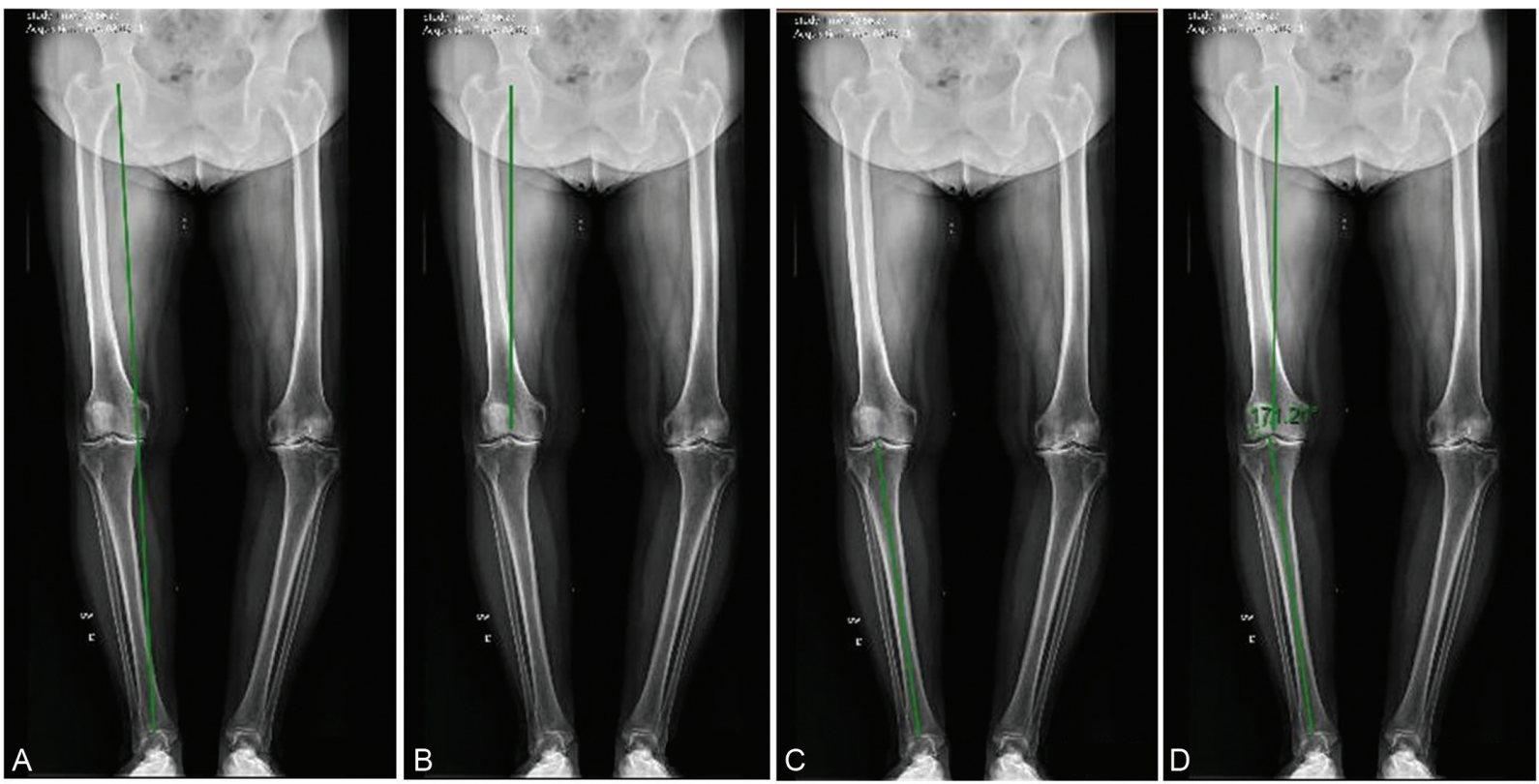

Fig. 1 Radiografias panorâmicas apresentando: (A) eixo mecânico do membro; (B) eixo mecânico do fêmur; (C) eixo mecânico da tíbia; (D) ângulo femorotibial- AFT.

submetidos à ATJ. Os critérios de inclusão contemplaram pacientes apresentando gonartrose primária como causa da cirurgia e classificados, conforme a classificação de Ahlback, ${ }^{8}$ entre os estágios de 3 a 5 . Os critérios de exclusão foram imagens radiológicas com baixa qualidade e pacientes longilíneos, cuja radiografia panorâmica não incluiu as articulações do quadril, joelho e tornozelo na mesma imagem.

Todos os 70 pacientes realizaram radiografias longas em apoio bipodal quando ainda estavam internados em nosso hospital durante o pós-operatório de ATJ. As radiografias foram digitalizadas e observadas através do programa de imagem PixvieweR (PixvieweR Web Solutions, Bronx, NY, EUA). As radiografias curtas do joelho foram confeccionadas a partir das radiografias longas, usando a linha articular como referência e recortando a imagem 10 centímetros acima e 10 centímetros abaixo dela, conforme o estudo prévio de Ishii et al. $^{9}$ Dessa forma, evitamos a influência de possíveis rotações oriundas da realização de uma nova radiografia.

As radiografias foram realizadas no Centro de Diagnóstico por Imagem de nossa instituição. As 140 imagens foram analisadas ao acaso, em momentos diferentes, por três ortopedistas generalistas. Todas as imagens foram reavaliadas pelos mesmos ortopedistas em um período de 15 dias. Em todas as radiografias, o eixo mecânico do membro, do fêmur e da tíbia foram traçados e os ângulos femorotibiais calculados ( - Figura 1).

O eixo mecânico do membro foi traçado por uma linha que passa através do centro da cabeça do fêmur ao centro do tornozelo (-Figura 1A). O eixo mecânico do fêmur foi definido como a linha que une o centro da cabeça do fêmur ao ápice do intercôndilo femoral (-Figura 1B). Já o eixo mecânico da tíbia foi definido como uma linha entre o centro do planalto tibial e o centro do tálus (-Figura 1C). A mensuração do ângulo-femorotibial (AFT) foi realizada a partir dos eixos mecânicos do fêmur e da tíbia (- Figura 1D). Na radiografia curta do joelho, como não é possível visualizar as imagens do quadril e do tornozelo, foi traçada uma imagem inferindo a localização desses pontos para traçar o eixo mecânico (—Figura 2).

A análise estatística foi realizada utilizando o software SPSS versão 13.0 (SPSS Inc., Chicago, IL, EUA). O coeficiente de correlação intraclasse (CCI) foi calculado para avaliar a concordância da medida do eixo mecânico inter e intraobservador. É sabido que quanto mais próximo o $\mathrm{CCl}$ for de 1 , mais forte é a concordância entre os observadores. Ao contrário, quanto mais próximo de 0 (zero), mais fraca se torna a concordância.

\section{Resultados}

Foi observado um ângulo femorotibial médio de 2,5 graus nas radiografias curtas, enquanto nas radiografias panorâmicas observamos um valor de 1,8 graus. Considerando-se cada examinador isoladamente, a medida do eixo mecânico variou no máximo 8 graus entre as radiografias longa e curta (-Figura 3). Após a análise estatística, observou-se que existe alta concordância intra e interobservador quando foram utilizadas as radiografias panorâmicas, apresentando CCI mínimo intraobservador de 0,89 e interobservador de 0,92 , equivalente a uma concordância fortíssima. Já nas radiografias curtas em incidência AP do joelho, o CCI intraobservador mínimo foi de 0,70 e máximo de 0,75 , evidenciando uma concordância moderada-boa. O CCI interobservador evidenciou valor considerado bom, de 0,80 (- Tabela 1).

\section{Discussão}

O presente estudo comparou a acurácia intra e interobservador da avaliação do eixo mecânico entre radiografias panorâmicas de membros inferiores e radiografias de joelho no pós-operatório de pacientes submetidos a ATJs realizadas no nosso serviço. As radiografias panorâmicas de membros 


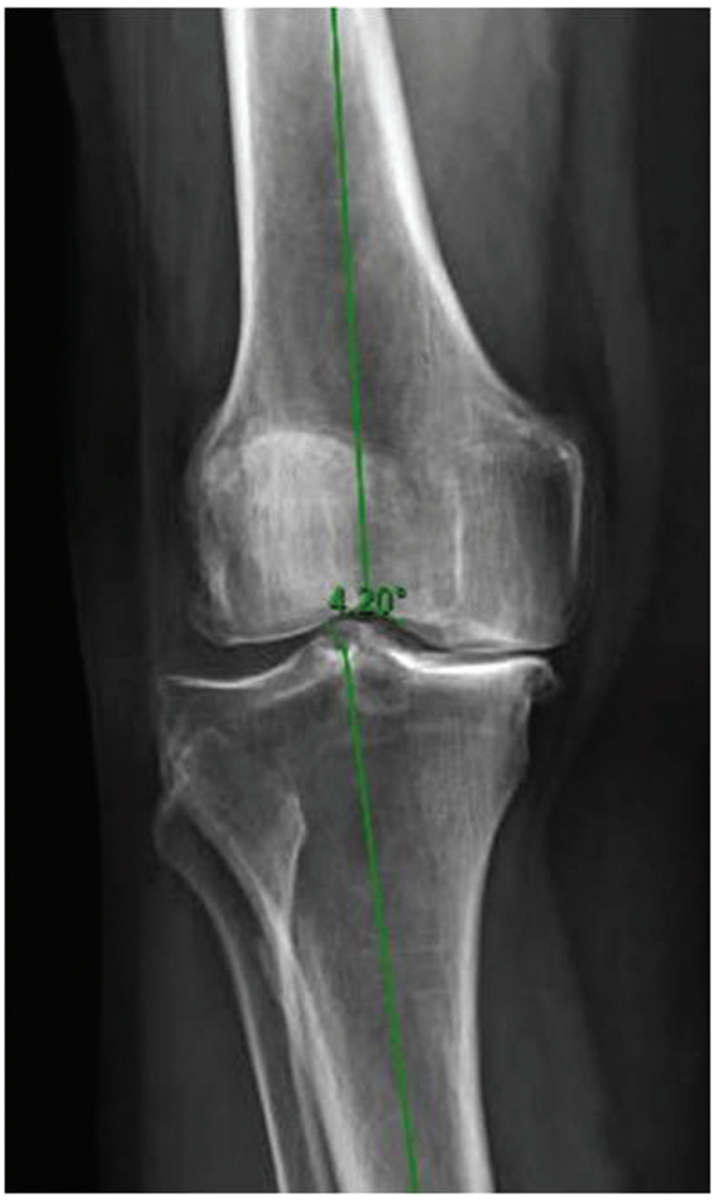

Fig. 2 Eixo mecânico femorotibial pré-operatório na radiografia curta.

Tabela 1 Valores obtidos para concordância intra e interobservador

\begin{tabular}{|l|l|l|}
\hline & & $\begin{array}{l}\text { Coeficiente de correlacão } \\
\text { intraclasse (CCI) }\end{array}$ \\
\hline Intraobservador & & Mecânico \\
\hline $\mathbf{1}$ & Longa & 0,90 \\
& Curta & 0,73 \\
\hline $\mathbf{2}$ & Longa & 0,89 \\
& Curta & 0,75 \\
\hline 3 & Longa & 0,92 \\
& Curta & 0,70 \\
\hline Interobservador & Longa & 0,92 \\
& Curta & 0,80 \\
\hline
\end{tabular}

Observações: $\mathrm{CCl} \leq 0,20$-sem concordância; $0,20<\mathrm{CCl} \leq 0,40$-concordância fraca; $0,40<\mathrm{CCl} \leq 0,60$-concordância moderada; $0,60<$ $\mathrm{CCl} \leq 0,80$-concordância forte (boa); $\mathrm{CCl}>0,80$-concordância fortíssima (ótima). Intervalo de confiança de $95 \%$ para $\mathrm{CCl} ; p<0,001$.

inferiores são classicamente utilizadas quando se quer analisar eixos mecânicos e anatômicos com precisão. ${ }^{10-12}$ Porém, é sabido que tais imagens radiográficas aumentam o custo real para a instituição, adicionam radiação desnecessária a órgãos pélvicos e requerem salas e equipamentos especiais para sua realização. ${ }^{2}$ Devido a essas limitações,
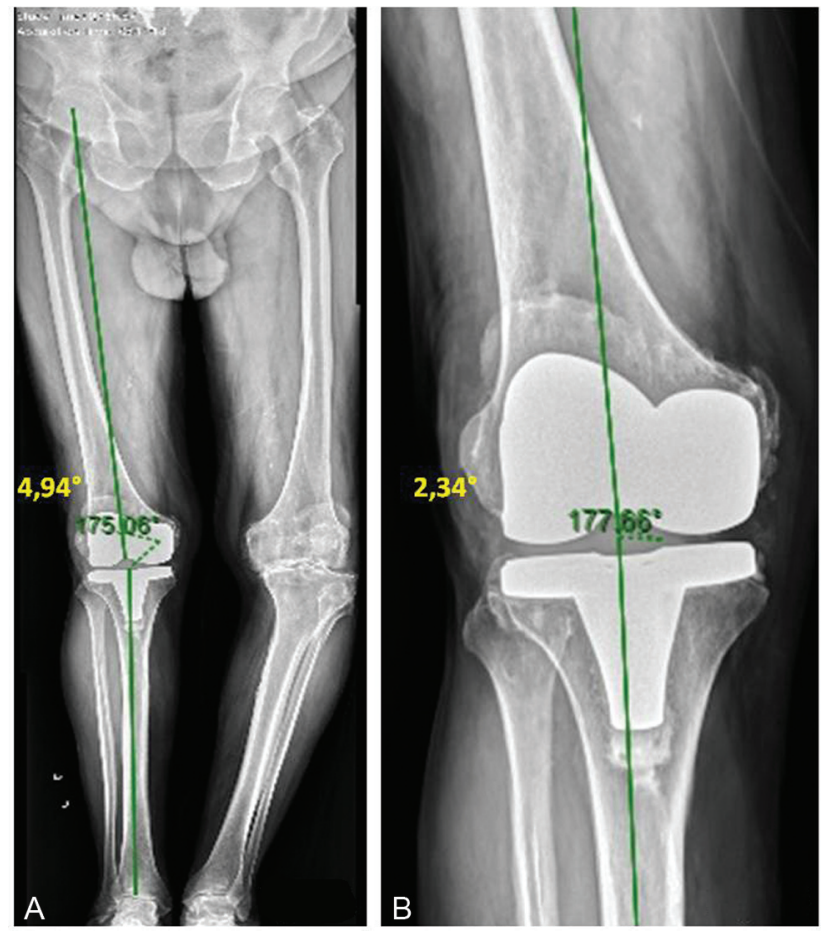

Fig. 3 Comparação do ângulo femorotibial nas radiografias pósoperatórias longa (A) e curta (B).

diversos estudos propuseram alternativas às radiografias longas para confeccionar um eixo mecânico confiável. ${ }^{9}$

Um dos estudos pioneiros na avaliação do eixo mecânico foi o de Abu-Rajab et al. ${ }^{5} \mathrm{O}$ grupo avaliou 50 radiografias consecutivas de pacientes em pós-operatório de ATJ e compararam os CCIs das radiografias panorâmicas e radiografias padronizadas do joelho. O CCI interobservador da radiografia dos membros inferiores foi de 0,95 , comparado a 0,51 quando avaliadas apenas radiografias de joelho. Dargel et al. ${ }^{13}$ obtiveram resultados similares aos do presente estudo, porém compararam os CCI de radiografias panorâmicas e radiografias cortadas em $80 \%$, $60 \%$ e $40 \%$ do comprimento total do membro inferior. Os autores encontraram CCI de 0,95 ao analisarem o eixo mecânico com a imagem panorâmica, comparado com 0,89 da radiografia em $80 \%, 0,76$ da radiografia em $60 \%$ e 0,61 da radiografia cortada em $40 \%$ do membro. Esses dados sugerem fortemente que a avaliação do eixo se torna mais acurada à medida que maiores segmentos dos membros inferiores são demonstrados na radiografia. O grupo concluiu que a radiografia do joelho é insuficiente para avaliar o eixo mecânico, sugerindo a realização de rotina do exame panorâmico no pós-operatório de ATJ.

$O$ presente estudo encontrou resultados semelhantes aos descritos na literatura internacional. Nossos resultados mostraram uma concordância fortíssima intra e interobservador das medidas dos eixos nas radiografias panorâmicas, o que vai de encontro aos trabalhos descritos previamente., ${ }^{5,13}$ Além disso, demonstramos que as radiografias curtas foram inferiores na capacidade de avaliar de modo acurado o alinhamento coronal do membro inferior, visto que mostraram um nível intra e interobservador apenas moderado.

Uma das críticas em relação aos estudos de avaliação do alinhamento consiste em ser realizado em apenas um plano, 
já que as artroplastias fazem parte de um procedimento tridimensional. Chauhan et al. ${ }^{14}$ utilizaram avaliação tomográfica em 12 joelhos operados de 6 cadáveres utilizando o protocolo de Perth. Foram encontrados seis parâmetros posicionais dos componentes que, individualmente ou somados, podem interferir nos resultados clínicos dos pacientes. São eles as posições dos componentes femoral e tibial em flexão ou extensão, em varo ou valgo, em rotação externa ou interna e, por fim, a má seleção dos tamanhos dos componentes. Apesar das inúmeras informações que a tomografia computadorizada pode fornecer, é importante destacar que a mesma não é utilizada de rotina no pós-operatório, pois, além de não ser um exame de fácil acesso no nosso país pelo seu elevado custo, ainda aumenta significativamente a radiação à qual o paciente é submetido.

Entre os aspectos positivos do presente estudo, cabe destacar que este é o primeiro trabalho nacional avaliando o alinhamento mecânico no pós-operatório de ATJs. Ainda, os nossos resultados vão de encontro aos publicados na literatura internacional e com amostra semelhante. Entre suas limitações, destacamos que a avaliação do alinhamento pósoperatório foi realizada apenas no plano coronal, enquanto sabemos que um bom funcionamento da ATJ necessita de equilíbrio e bom posicionamento nos três planos. Além disso, outros indicadores significativos de resultados clínicos, tais como avaliação dos scores de dor no pré e pós-operatório e avaliação das complicações, não foram aferidos.

\section{Conclusão}

As radiografias panorâmicas do joelho são mais acuradas para avaliar o eixo mecânico do membro no pós-operatório de pacientes submetidos à ATJ. Seguindo os estudos internacionais previamente realizados, sugerimos a inclusão da radiografia panorâmica na rotina do pós-operatório deste procedimento.

\section{Suporte Financeiro}

Não houve suporte financeiro de fontes públicas, comerciais, ou sem fins lucrativos.

Conflito de Interesses

Os autores declaram não haver conflito de interesses.

\section{Referências}

1 Fernandes DA, Poeta LS, Martins CAQ, Lima F, Rosa Neto F. Equilíbrio e qualidade de vida após artroplastia total de joelho. Rev Bras Ortop 2018;53(06):747-753

2 Carvalho Júnior LH, Castro CAC, Gonçalves MBJ, Rodrigues LCM, Lopes FL, Cunha FVP. Complicações de curto prazo da artrolastia total de joelho: avaliação de 120 casos. Rev Bras Ortop 2006;41 (05):162-166

3 Fang DM, Ritter MA, Davis KE. Coronal alignment in total knee arthroplasty: just how important is it? J Arthroplasty 2009;24(6, Suppl)39-43

4 Lotke PA, Ecker ML. Influence of positioning of prosthesis in total knee replacement. J Bone Joint Surg Am 1977;59(01): 77-79

5 Abu-Rajab RB, Deakin AH, Kandasami M, McGlynn J, Picard F, Kinninmonth AW. Hip-Knee-Ankle Radiographs Are More Appropriate for Assessment of Post-Operative Mechanical Alignment of Total Knee Arthroplasties than Standard AP Knee Radiographs. J Arthroplasty 2015;30(04):695-700

6 Felson DT, Cooke TD, Niu J, et al; OAI Investigators Group. Can anatomic alignment measured from a knee radiograph substitute for mechanical alignment from full limb films? Osteoarthritis Cartilage 2009;17(11):1448-1452

7 Sheehy L, Felson D, Zhang Y, et al. Does measurement of the anatomic axis consistently predict hip-knee-ankle angle (HKA) for knee alignment studies in osteoarthritis? Analysis of long limb radiographs from the multicenter osteoarthritis (MOST) study. Osteoarthritis Cartilage 2011;19(01):58-64

8 Alhbäck S. Osteoarthritis of the knee. A radiographic investigation. Acta Radiol Diagn (Stockh) 1968(Suppl 277):7-72

9 Ishii Y, Ohmori G, Bechtold JE, Sherman RE, Gustilo RB. Accuracy of the short radiograph in the measurement of the tibiofemoral angle. Knee 1995;2(02):81-84

10 Maquet PG. Biomechanics of the knee. New York: Springer-Verlag; 1976

11 Moreland JR, Bassett LW, Hanker GJ. Radiographic analysis of the axial alignment of the lower extremity. J Bone Joint Surg Am 1987;69(05):745-749

12 Sharma L, Song J, Felson DT, Cahue S, Shamiyeh E, Dunlop DD. The role of knee alignment in disease progression and functional decline in knee osteoarthritis. JAMA 2001;286(02): 188-195

13 Dargel J, Pennig L, Schnurr C, Boese CK, Eysel P, Oppermann J. [Should we use hip-ankle radiographs to assess the coronal alignment after total knee arthroplasty?] Orthopade 2016;45(07):591-596

14 Chauhan SK, Clark GW, Lloyd S, Scott RG, Breidahl W, Sikorski JM. Computer-assisted total knee replacement. A controlled cadaver study using a multi-parameter quantitative CT assessment of alignment (the Perth CT Protocol). J Bone Joint Surg Br 2004;86 (06):818-823 